\title{
Negative Impact of Statins on Oligodendrocytes and Myelin Formation In Vitro and In Vivo
}

\author{
Steve Klopfleisch, ${ }^{1 \star}$ Doron Merkler, ${ }^{2 \star}$ Matthias Schmitz, ${ }^{1}$ Sabine Klöppner, ${ }^{1}$ Mariann Schedensack, ${ }^{2}$ Gunnar Jeserich, ${ }^{4}$ \\ Hans H. Althaus, ${ }^{1}$ and Wolfgang Brück ${ }^{2,3}$ \\ ${ }^{1}$ Max Planck Institute for Experimental Medicine, 37075 Göttingen, Germany, ${ }^{2}$ Department of Neuropathology, University Medical Centre, Georg August \\ University, 37075 Göttingen, Germany, ${ }^{3}$ Institute for Multiple Sclerosis Research, Gemeinnützige Hertie-Stiftung and University Medical Centre Göttingen, \\ 37073 Göttingen, Germany, and ${ }^{4}$ Department of Neurobiology, University of Osnabrück, 49076 Osnabrück, Germany
}

\begin{abstract}
Statins are widely prescribed drugs in cardiovascular diseases. Recent studies also demonstrated anti-inflammatory and immunomodulatory properties of statins by modulating the activity of small GTPases. Statins are thus considered as potential therapeutic drug for the inflammatory demyelinating disease multiple sclerosis (MS). However, little is known about the effects of statins on myelin-forming oligodendrocytes. Here, we show that statins hamper process and myelin formation in vitro by interfering with Ras and Rho signaling in mature oligodendrocytes and provide evidence that statins impair ongoing remyelination in vivo. Our findings may have significant implications for the application of statins in MS patients and in other demyelinating diseases of the CNS.
\end{abstract}

Key words: remyelination; multiple sclerosis; statins; cuprizone; oligodendrocytes; GTPases

\section{Introduction}

Statins (or HMG-CoA reductase inhibitors) are classically used to lower cholesterol levels in people with or at risk of cardiovascular disease and are prescribed in $>25$ million patients worldwide (Topol, 2004). However, due to its potential salutary effects in a wide range of diseases, including multiple sclerosis (MS), neurodegenerative disorders, nonischemic cardiomyopathy or even some types of cancers, it was suggested that statins exert pleiotropic effects beyond cholesterol lowering (Liao and Laufs, 2005). Most was attributed to anti-inflammatory and immunomodulatory effects mainly via modulating the activity of small GTPases involving Ras and Rho (Greenwood et al., 2006). Accordingly, statins have been reported to beneficially modulate the immune response in experimental autoimmune encephalomyelitis (EAE), an animal model of MS (Youssef et al., 2002). Hence, statins are currently evaluated as potential new drugs for MS treatment (Sena et al., 2003; Vollmer et al., 2004). Disseminated demyelinated lesions within the CNS are the hallmark of MS. Nevertheless, a considerable portion of MS lesions shows signs of new myelin formation (remyelination) that may depend on recruitment of oligodendrocyte precursors and growth factor signaling on mature oligodendrocytes (Gensert and Goldman, 1997; Franklin, 2002; Althaus, 2004). In line with this assumption, it was documented that statins may induce a proremyelinating environment that resulted in augmented remyelination in EAE (Paintlia et al., 2005). Nevertheless, the direct

\footnotetext{
Received June 17, 2008; revised 0ct. 24, 2008; accepted Nov. 5, 2008

This work was supported by grants from the Gemeinnützige Hertie-Stiftung to H.H.A. and W.B. We thank B. Maruschak for expert technical assistance.

*S.K. and D.M. contributed equally to this work.

Correspondence should be addressed to Doron Merkler at the above address.E-mail:merkler@med.uni-goettingen.de. DOI:10.1523/JNEUROSCI.2765-08.2008

Copyright $\odot 2008$ Society for Neuroscience $\quad 0270-6474 / 08 / 2813609-06 \$ 15.00 / 0$
}

impact of statins on resident cells within the CNS is difficult to address in the EAE model since the neuroinflammatory response is also reduced by this treatment. Furthermore, it was recently noted that statins caused retraction of oligodendroglial processes in vitro (Miron et al., 2007). In the present work, we delineate the molecular impact of statins on oligodendrocytes with regard to process formation and myelin synthesis in vitro. Furthermore, we provide evidences that statins negatively influence spontaneous remyelination in vivo in the mouse model of cuprizone-induced demyelination.

\section{Materials and Methods}

Cell culture. Oligodendrocytes (OLs) were isolated from adult pig brains, cultured on poly-D-lysine-coated Petri dishes or multiwell culture plates and immunocytochemically characterized (data not shown). After $6 \mathrm{~d}$ in vitro, culture medium was removed and replaced by fresh culture medium containing $1 \%$ of a special low-cholesterol serum (fetal calf serum GOLD, PAA). Statin simvastatin (Sst, $5 \mu \mathrm{M}$ ) was added (Hydroxy-Sst, Calbiochem) followed by incubation time either 48,72 or $96 \mathrm{~h}$ as indicated. In a subset of experiments cells were also incubated with nerve growth factor (NGF, $50 \mathrm{ng} / \mathrm{ml}$, Sigma) or the intermediates mevalonate (200 $\mu \mathrm{M}$, Fluka) and geranylgeranylpyrophosphate (GGPP, $10 \mu \mathrm{M}$, Sigma). Farnesylpyrophosphate (FPP, $10 \mu \mathrm{M}$, Sigma) was transferred into OLs by transfection using jetSI-ENDO reagent (Biomol). Transfection reagent only served as a control.

Immunoblot assay of p21Ras and RhoA, p21Ras, RhoA- and ROCKactivity. OLs were pretreated with $1 \mathrm{~mm}$ sodium orthovanadate (Sigma) for $1 \mathrm{~h}$ and harvested by scraping on ice. After pelleting cells and washing in PBS they were dissolved in $10 \mathrm{~mm}$ Tris- $\mathrm{HCl}$ buffer, $\mathrm{pH}$ 7.4, containing inhibitors $1 \mathrm{~mm}$ sodium orthovanadate, $1 \mathrm{~mm}$ sodium fluoride (Sigma), $1 \mathrm{~mm}$ phenylmethylsulfonyl fluoride (Merck), $1 \mu \mathrm{g} / \mathrm{ml}$ aprotinin (Sigma) and $1 \mu \mathrm{g} / \mathrm{ml}$ leupeptin (Sigma) and lysed by sonification. Membrane and cytosolic fractions were separated by centrifugation at 100,000 $\times g$ and $4^{\circ} \mathrm{C}$ for $45 \mathrm{~min}$. After dissolving both fractions in $2 \%$ SDS containing all inhibitors, protein concentrations were measured using the DC-protein- 
assay (Bio-Rad). The same amounts of protein were mixed with sample buffer, heated at $95^{\circ} \mathrm{C}$ for $5 \mathrm{~min}$ and loaded on $15 \%$ SDS-gels and separated by PAGE. After transferring proteins to nitrocellulose membrane (GE Healthcare), blots were blocked with PBS containing 5\% dry milk, $0.5 \%$ Tween 20 for $2 \mathrm{~h}$ at room temperature and probed overnight at $4{ }^{\circ} \mathrm{C}$ with anti-pan-Ras (Ab-3) (Calbiochem, 1:100) or anti-RhoA (Santa Cruz Biotechnology, 1:100). Blots were washed in 0.5\% Tween 20/PBS and incubated with HRP-conjugated secondary anti-mouse antibody (GE Healthcare, 1:2000). Bands were visualized by the enhanced chemiluminescence (ECL) detection system (Amersham) according to the manufacturer's instructions and exposure to $\mathrm{x}$-ray film. Activated form of p21Ras (p21Ras-GTP) and RhoA (Rho-GTP) were measured with the Ras or Rho Activation Assay Kit (Upstate), respectively, according to the protocols provided by the manufacture. ROCK activity was determined according to the protocol provided by the manufacturer (Biozol).

Mitogen-activated protein kinase-in-gel-kinase-assay. Radioactive mitogen-activated protein kinase (MAPK) assay was performed according to the protocol published previously (Althaus et al., 1997). Briefly, protein concentrations from cell lysates were determined using the DCprotein assay (Bio-Rad) and identical protein amounts of different tested conditions as indicated were separated on a myelin basic protein (MBP) containing SDS gel. Radioactive labeled ATP was incorporated into MBP by active MAPK Erk1/2. Bands of incorporated ATP were visualized by autoradiography, using x-ray film.

Cuprizone feeding. All studies were performed in accordance with the German animal protection laws and approved by the responsible governmental authority (Bezirksregierung Braunschweig).

A total of 152 female C57BL/ 6 mice, 7 to 8 weeks old, were purchased from Charles River.

Animals were fed with $0.2 \%(\mathrm{w} / \mathrm{w})$ cuprizone (Sigma) in ground breeder chow ad libitum for 6 weeks or left under normal diet (agematched controls). At day 0 after cuprizone removal, four animals of the cuprizone-treated group and four age-matched untreated controls were killed to verify demyelination by light microscopy. Directly after cuprizone removal animals were randomly assigned to the following treatment groups: (a) control treatment (diluent only), (b) $1 \mathrm{mg} \mathrm{Sst} / \mathrm{kg}$ b.wt., (c) $10 \mathrm{mg} \mathrm{Sst} / \mathrm{kg}$ b.wt., (d) $50 \mathrm{mg} \mathrm{Sst} / \mathrm{kg}$ b.wt. One, two, or four weeks after cuprizone removal, six animals from each treatment group (a-d) were killed for light microscopical analysis. A subset of animals were fed with $0.2 \%(\mathrm{w} / \mathrm{w})$ cuprizone in ground breeder chow ad libitum for 6 weeks or left under normal diet (age-matched controls). At day 0 after cuprizone removal animals were randomly assigned into groups as follows: ( $\left.\mathrm{a}^{\prime}\right)$ control treatment (diluent only), ( $\left.\mathrm{b}^{\prime}\right) 10 \mathrm{mg}$ Sst/kg b.wt., and (c') $50 \mathrm{mg} \mathrm{Sst} / \mathrm{kg}$ b.wt. Three or six weeks after cuprizone removal, three animals from each treatment group $\left(\mathrm{a}^{\prime}-\mathrm{c}^{\prime}\right)$ were killed for electron microscopical analysis.

Tissue preservation and staining. For histological analysis, animals received an overdose of ketamine/metomidine and were perfused transcardially with either $4 \%$ paraformaldehyde for light microscopy or $4 \%$ glutaraldehyde for electron microscopy and removed brains were postfixed in corresponding fixative overnight. Segments of brains were prepared for paraffin or electron microscopy from corpus callosum. Paraffin sections were stained with Luxol Fast Blue (LFB), periodic acid-Schiff (PAS). For electron microscopy, sections from epon-embedded corpus callosum were cut and stained with toluidine blue. The tissue was then trimmed and reoriented so that ultrathin cross sections of midline corpus callosum could be cut and treated with uranyl acetate and lead citrate.

Light and electron microscopic analysis. Demyelination was scored ranging from 0 (no demyelination) to 3 (complete demyelination) on LFB/PAS paraffin-embedded sections as described previously (Hiremath et al., 1998). Electron micrographs were analyzed using the analysis DocuSystem (Soft Imaging System). At least 800 fibers from each mouse $(n=3$ per time point and treatment) were analyzed. G-ratios were defined as diameter of the axon divided by fiber diameter (i.e., axon and myelin). In addition, the number of nonmyelinated fibers in relation to all fibers was counted and expressed as percentage nonmyelination.

Sst treatment of mice. After withdrawal of cuprizone, animals were treated with 1, 10, or $50 \mathrm{mg} \mathrm{Sst/kg} \mathrm{b.w.} \mathrm{Sst} \mathrm{suspensions} \mathrm{were} \mathrm{prepared} \mathrm{by}$ mixing Sst in a $0.2 \%$ agarose gel. The suspension was homogenized and tempered at $37^{\circ} \mathrm{C}$. Controls were prepared by application by tempering $0.2 \%$ agarose gel. Treatment was given once daily over indicated time periods via a pharyngeal tube.

Brain lysates for Western blot analysis of expressed myelin proteins. Freshly prepared brains were cut and snap frozen in liquid nitrogen and stored at $-80^{\circ} \mathrm{C}$ until further processing. Corpus callosum tissue was dissected and homogenized in $10 \mathrm{~mm}$ Tris-HCl-buffer, $\mathrm{pH}$ 7.4, and suspension was sonificated on ice. Brain homogenate $(200 \mu \mathrm{l})$ was mixed with $50 \mu \mathrm{l}$ of a $10 \%$ SDS solution. Suspension was centrifuged at 10,000 $g$ for $25 \mathrm{~min}$ at $4^{\circ} \mathrm{C}$ and supernatant was collected. Protein concentrations were measured using the DC protein assay (Bio-Rad). After mixing with sample buffer and heating at $95_{i} \mathrm{C}$ for $2 \mathrm{~min}$, defined amounts of proteins were separated on SDS gels [ $1 \mu \mathrm{g}$ for MBP, $5 \mu \mathrm{g}$ for proteolipidprotein (PLP) and $20 \mu \mathrm{g}$ for $2^{\prime}, 3^{\prime}$-cyclic nucleotide $3^{\prime}$-phosphodiesterase (CNP)]. After Western blotting, membranes were blocked and incubated with primary antibody [MBP, Santa Cruz Biotechnology, 1:3000; PLP, provided by K.A. Nave (Max Planck Institute for Experimental Medicine, Göttingen, Germany), 1:100; CNP, Sigma, 1:500], washed and incubated with secondary antibodies. Proteins were visualized using the ECL detection system (GE Healthcare) and x-ray film.

Detection of newly synthesized myelin proteins. Cells were incubated with $20 \mu \mathrm{M}$ radioactive labeled leucine $\left({ }^{14} \mathrm{C}\right.$-leucine, GE Healthcare) for different times. After removing cells by scraping they were pelleted and dissolved in $1 \%$ SDS. Protein concentrations were measured using the DC protein assay (Bio-Rad). Sample buffer was added before heating the probes at $95^{\circ} \mathrm{C}$ for $5 \mathrm{~min}$. The same protein amounts were separated on a $12 \%$ SDS gel. As a reference, myelin was isolated from pig brain and separated in the gel. Afterward, the gel was fixed for $1 \mathrm{~h}$ in $30 \%$ methanol (Baker)/10\% acetic acid (Merck) and incubated for $1 \mathrm{~h}$ in an enhancer (EN3HANCE ${ }^{\mathrm{a}}$, DuPont). Newly synthesized proteins were visualized by autoradiography.

Statistics. Analyses were performed with GraphPad Prism software (version 4.0). To assess significant differences between two groups, Student's $t$ tests were applied, whereas differences between single measurements of $>2$ groups were assessed by one-way ANOVA followed by least significant difference post-test if the $F$ test of ANOVA indicated statistically significant differences. A $p$ value $<0.05$ was considered significant, and a $p<0.01$ was considered highly significant.

\section{Results}

Interference of Ras- and Rho-signaling by simvastatin causes retraction of oligodendroglial processes in vitro

In a recent study, it has been shown that statins can induce retraction of oligodendroglial processes and decrease cell survival in vitro (Miron et al., 2007). Furthermore, it was noted that the detrimental outcome of oligodendrocytes could be prevented by coincubation with several intermediates of cholesterol biosynthesis (supplemental Fig. 1, available at www.jneurosci.org as supplemental material) ruling out a direct toxic effect of statins. Mechanistically, the extension of oligodendroglial processes is linked to the p21Ras/MAPK(Erk) pathway and Rho signaling (Stariha et al., 1997; Liang et al., 2004). Proper membrane targeting of p21Ras and Rho is essential for activation and signaling and depends on the prenylation by intermediates of cholesterol biosynthesis such as FPP and GGPP, respectively (Casey, 1995). We therefore analyzed whether treatment with the Sst causes reduction of these intermediates in oligodendrocytes in vitro and thus could interfere with the corresponding signaling pathways. In a first set of experiments, we assessed the impact of Sst on membrane anchoring of oligodendroglial p21Ras in oligodendrocytes. For this purpose, membrane and cytosolic fractions of p21Ras were determined by Western blot analysis (Fig. 1A). Treatment of oligodendrocytes with Sst reduced membrane-bound p21Ras and increased cytosolic p21Ras. Addition of either cholesterol biosynthesis intermediates mevalonate (that can be metabolized to FPP) or FPP itself completely reversed this translocation of 
A
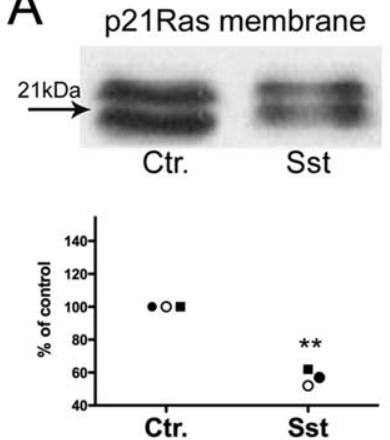
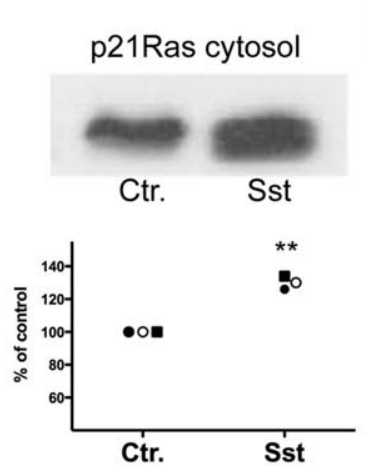
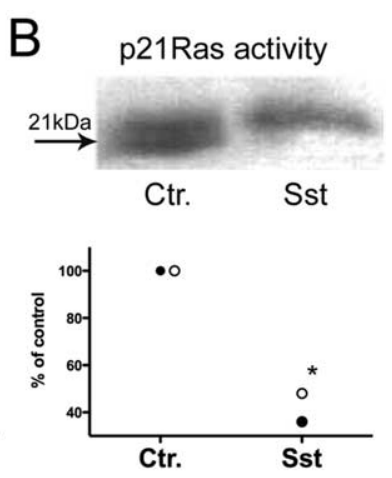
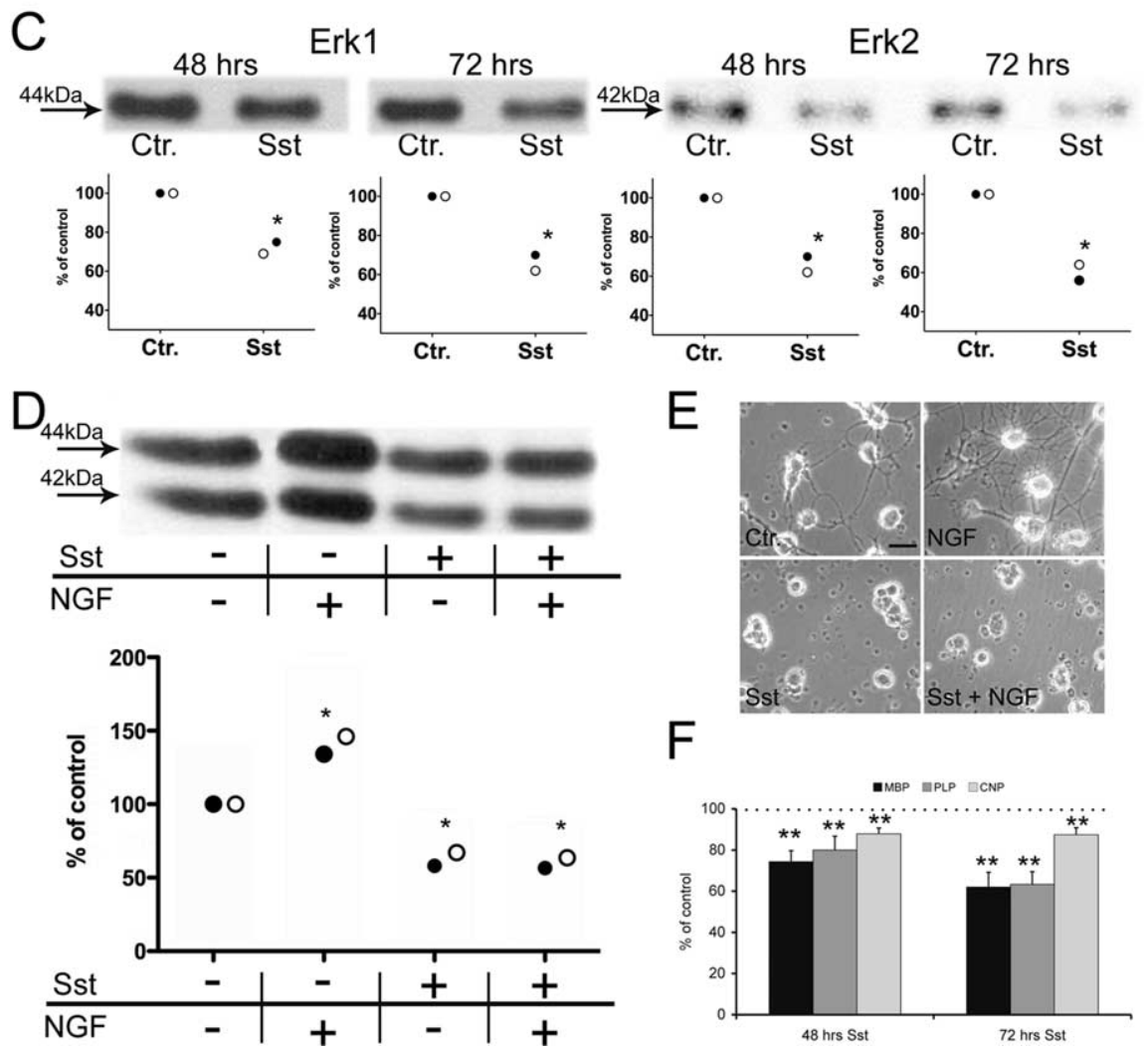

$\mathrm{F}$

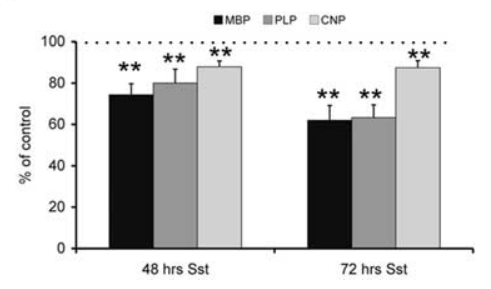

Figure 1. Simvasatin (Sst) reduces p21Ras membrane association, Erk1/2 activity and myelinogenesis of oligodendrocytes in vitro. $\boldsymbol{A}$, Distribution of membrane (left) and cytosolic (right) fraction of p21Ras were quantified by Western blot analysis (signal of control "Ctr." was set as 100\%). Sst treatment significantly reduced membrane-bound p21Ras and increased the cytosolic p21Ras compared with untreated controls. B, Decreased Ras activity was revealed in the p21Ras pull-down assay. $\boldsymbol{C}$, Sst reduced downstream activity of MAPK Erk1 (left two panels) and Erk2 (right two panels) after $48 \mathrm{~h}$ and after $72 \mathrm{~h}$. D, Reduction of Erk1/2 activity by Sst could not be prevented by the addition of NGF. $\boldsymbol{E}$, Accordingly, enhanced oligodendroglial process formation was observable if NGF was added solely (top right) but not simultaneously with Sst (bottom right). $\boldsymbol{F}$, Measurement of ${ }^{14} \mathrm{C}$-leucine incorporation of the myelin proteins MBP, PLP and CNP revealed reduced myelinogenesis after exposure to Sst for $72 \mathrm{~h}$ (signal of control "Ctr." was set as 100\%). Each of the symbols (closed circle, open circle or square) in panels $\boldsymbol{A}-\boldsymbol{D}$ represents the mean of one independent experiment (consisting of 5- 6 replicates per group). Results in $\boldsymbol{F}$ are presented as mean + SEM of three independent experiments. Total protein concentration was determined as indicated in material and methods and total protein amount was adapted to equal the amount for each tested sample. Statistical analyses were performed for control versus treatment group(s). ${ }^{*} p<0.05,{ }^{* *} p<0.01$. Scale bar, $10 \mu \mathrm{m}$.

p21Ras (supplemental Fig. 2, available at www.jneurosci.org as supplemental material). Furthermore, GTP-bound p21Ras (the activated form) was reduced by $\sim 60 \%$ as determined in pulldown assays (Fig. $1 B$ ). Subsequently, we determined the effect of p21Ras translocation on the downstream effectors MAPK(Erk1/ 2). Erk1/2 activities were both significantly reduced in oligodendrocytes after exposure to Sst and showed only $\sim 65 \%$ of control activity (Fig. 1C). Erk1/2 activities are induced upon exposure to NGF in oligodendrocytes (Althaus et al., 1997). Accordingly,

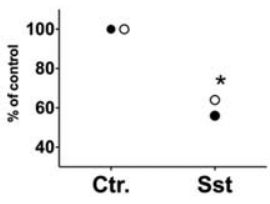

NGF signaling via TrkA/p21Ras resulted in enhanced process formation (Fig. $1 E$ ). Since the p21Ras/MAPK pathway was impaired by Sst, addition of NGF could neither reverse the reduced Erk1/2 activity (Fig. 1D) nor enhance oligodendroglial process formation (Fig. $1 E$ ). Furthermore, activation of Erk1/2 was shown to induce myelinogenesis (Bhat and Zhang, 1996). We therefore tested whether Sst interferes with the synthesis of myelin proteins such as MBP, PLP or CNP in vitro. Measurement of ${ }^{14} \mathrm{C}$-leucine incorporation revealed a significant reduction of all three myelin proteins upon Sst exposure (Fig. $1 F)$. In addition, we investigated the effect of Sst on the Rho signaling cascade which was shown to be associated with cytoskeletal rearrangements, such as retraction of cellular processes (Riento and Ridley, 2003). Sst treatment caused translocation of membrane bound Rho to the cytosol (Fig. 2A). Treatment of OLs with Sst caused a reduction of membrane-bound RhoA by $\sim 60 \%$ and an increase of cytosolic RhoA by $\sim 150 \%$ compared with untreated control. Interestingly, the activity of RhoA (170\%) and its effector ROCK $(150 \%)$ was strongly induced compared with untreated control (Figs. 2B,C). Although the reason for this latter unexpected outcome remains to be investigated in the future, it is consistent with earlier studies showing that pharmacological inhibition of ROCK activity results in enhanced oligodendroglial process formation (Riento and Ridley, 2003).

\section{Intrinsic remyelination is delayed by statins in vivo}

We further analyzed the influence of Sst on spontaneous remyelination after a demyelinating insult in vivo. Cuprizone feeding induces consistent demyelination within the corpus callosum of C57BL/6 mice followed by substantial spontaneous remyelination within a few weeks of cuprizone withdrawal (Matsushima and Morell, 2001). We applied Sst daily perorally at 1 $\mathrm{mg} / \mathrm{kg}$ b.w., $10 \mathrm{mg} / \mathrm{kg}$ b.w. or $50 \mathrm{mg} / \mathrm{kg}$ b.w. Animals treated with various doses of Sst following cuprizone challenge revealed impaired remyelination compared with untreated controls (Fig. 3A). Doubleblind analysis of tissue sections demonstrated the negative impact of Sst, even for the lowest dose of $1 \mathrm{mg} / \mathrm{kg}$ b.w., on remyelination within the corpus callosum $(p<0.05)$ (Fig. $3 B)$. We further assessed remyelination by electron microscopy in a subgroup of animals (Fig. 3C-E). The degree of remyelination for both control and Sst-treated animals was determined by the percentage of unmyelinated axons and by the g-ratio 3 and 6 weeks after termination of cuprizone diet (Fig. $3 D, E$ ). Age-matched healthy control animals showed $4 \pm 1 \%$ (mean \pm SEM) unmyelinated axons, 


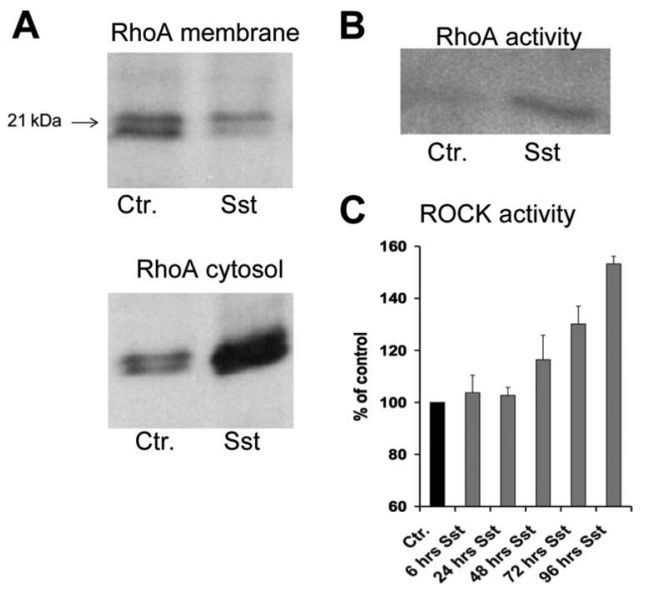

Figure 2. Sst reduces membrane association of RhoA but induces activity of RhoA and activity of the Rho-effector ROCK in oligodendrocytes in vitro. $A$, Treatment of OLs with Sst caused a reduction of membrane-bound RhoA by $\sim 60 \%$ and an increase of cytosolic RhoA by $\sim 150 \%$ compared with control (Western blot signal of control "Ctr." was set as 100\%). B, Of note, exposure to Sst induced RhoA-activity by $\sim 170 \%$ compared with control as evidenced by a Rho-activity assay. C, Similarly, activity of the Rho effector ROCK (Rho-kinase) was increased after Sst treatment to $150 \%$ of control level. Panels represent the summary of three independent experiments (each consists of $5-6$ replicates per group). Total protein concentration was determined as indicated in material and methods and total protein amount was adapted to equal the amount for each tested sample.

whereas after 6 weeks of cuprizone diet $\sim 66 \pm 5 \%$ of axons were unmyelinated. After 3 weeks of recovery, $34 \pm 5 \%$ of axons remained unmyelinated in untreated controls compared with $48 \pm$ $5 \%$ (10 mg Sst $/ \mathrm{kg}$ b.w.) and $48 \pm 3 \%$ (50 mg Sst $/ \mathrm{kg}$ b.w.) in Sst-treated animals ( $p<0.05$ Sst-treated versus control). This difference was even more accentuated 6 weeks after recovery. Control animals displayed an almost baseline level with $11 \pm 2 \%$ of unmyelinated fibers, whereas Sst-treated animals showed $42 \%$ (10 mg Sst/kg b.w.) or $44 \%$ (50 mg Sst $/ \mathrm{kg}$ b.w.) unmyelinated axons, respectively $(p<0.01 \%)$. The g-ratio (axon diameter to fiber diameter) was calculated as a further parameter for the assessment of remyelination (Coetzee et al., 1996). Untreated animals showed a g-ratio of $0.79 \pm 0.01$ (mean $\pm \mathrm{SD}$ ) which was increased to $0.93 \pm 0.02$ after 6 weeks of cuprizone diet. It returned to near normal values $(0.82 \pm 0.02)$ after 6 weeks of normal diet. In contrast, the g-ratio was significantly higher 3 and 6 weeks after recovery in Sst-treated animals [after 6 weeks $(0.89 \pm$ 0.01 ) than in spontaneously remyelinating controls $(p<0.05$ for both time points and doses)]. We further measured the amount of myelin by quantitative Western blot analysis for MBP, PLP and CNP from brain homogenates. Six weeks of cuprizone treatment induced a reduction of $30 \%$ for MBP, $54 \%$ for PLP and 50\% for $\mathrm{CNP}$ compared with age-matched healthy controls (supplemental Fig. 3A, available at www.jneurosci.org as supplemental material). Even 6 weeks after recovery, animals treated with $10 \mathrm{mg}$ Sst/kg b.w., showed significant reduction by $35 \%$ for MBP, $52 \%$ for PLP and 52\% for CNP compared with spontaneous remyeli- nating animals (supplemental Fig. 3B, available at www. jneurosci.org as supplemental material). Similar results were obtained with $50 \mathrm{mg} \mathrm{Sst} / \mathrm{kg}$ b.w. No differences in macrophages/ microglia infiltration was evident between the different treatments after cuprizone recovery (data not shown).

\section{Discussion}

Numerous studies have shown that statins target several key elements in the immunological cascade involved in inflammation and tissue damage (Liao and Laufs, 2005; Greenwood et al., 2006), and thus appear to be promising candidates for novel therapeutic drugs in MS. Nevertheless, the disease course in MS is characterized by broad interindividual variability with regard to the severity of symptoms during single MS attacks as well as to the extent of functional recovery: while some patients recover almost completely from an episode, others do not at all. The reason for this heterogeneity is still not understood, but it is conceivable that intrinsic regenerative capacities contribute to functional recovery during the remitting phase of MS (Franklin, 2002; Brück et al., 2003). Indeed, in a considerable fraction of MS cases, numerous demyelinating and remyelinating events occur during the decades-long disease course (Barkhof et al., 2003; Patrikios et al., 2006). Thus, in addition to the resolution of inflammation, remyelination seems to contribute to functional recovery in MS patients especially in the early phase of disease. Oligodendrocytes are highly metabolically active during myelin formation and any negative interference may result in failure of remyelination (Saher et al., 2005). Our data indicate that statin-induced modulation of Ras and Rho signaling is not only restricted to the immune compartment but also directly affects homeostasis of oligodendrocytes in vitro and in vivo. Previous studies indicated that statins may promote remyelination in the CNS in the EAE model (Paintlia et al., 2005, 2008). The discrepancy between our present results and previous findings might be explained by the different experimental systems used. Immunomodulatory effects mediated by statins cannot be easily differentiated from direct effects on resident cells of the CNS (as oligodendrocytes) in the EAE model, since a reduction of inflammation may always be accompanied by less destructive CNS lesions (an effect that we do not challenge by our study). In contrast, immunomodulatory effects are not as important in the cuprizone model as in the EAE model, since in the cuprizone model demyelination is primarily induced by toxic events (Blakemore, 1973; Ludwin, 1978). Therefore the cuprizone model is more suitable for studying effects directly related to oligodendrocytes but independent of inflammation. While we clearly acknowledge the potential beneficial effects of statins on inflammation in MS/EAE, our study nevertheless indicates that statins may negatively affect intrinsic properties of oligodendrocytes during remyelination, a process that may become particularly important during the recovery phase in MS when inflammation declines. Furthermore, protracted failure of remyelination as a consequence of statin treatment may also contribute to an increase in demyelination-associated axonal damage (Irvine and Blakemore, 2008). This may also affect axonal struc-

\footnotetext{
Figure 3. Simvastatin (Sst) treatment delays remyelination in vivo. A, LFB/PAS staining revealed extensive demyelination ("6 weeks cuprizone") that was still almost complete 1 week after recovery in all groups tested (top row, arrows indicate demyelinated corpus callosum, for comparison see "age-matched control"). Two and four weeks after recovery, Sst-treated animals (1, 10 or $50 \mathrm{mg} / \mathrm{kg}$ b.w., as indicated in $\boldsymbol{A}$ ) showed a delayed reestablishment of LFB staining compared with untreated controls (control). $\boldsymbol{B}$, The degree of demyelination on LFB/PAS-stained sections was scored on a scale of 0 (completely myelinated) to 3 (completely demyelinated). $\boldsymbol{C}$, Electron microscopy 3 or 6 weeks ( $n=3$ mice per group and time point) after recovery, respectively. Even after 6 weeks after curpizone removal, remyelination remained strongly impaired in Sst-treated animals. $\boldsymbol{D}$, The number of myelinated axons reached almost baseline levels 6 weeks after recovery in untreated controls, while in Sst-treated animals a substantial fraction of axons remained unmyelinated. $\boldsymbol{E}$, Similarly, g-ratio was significantly higher in Sst-treated animals 3 and 6 weeks after recovery. Results are presented as mean + SEM. ${ }^{*} p<0.05,{ }^{* *} p<0.01$. Scale bars: (in $\left.\boldsymbol{A}\right) 2 \mathrm{~mm}$; (in C) $1 \mu \mathrm{m}$.
} 


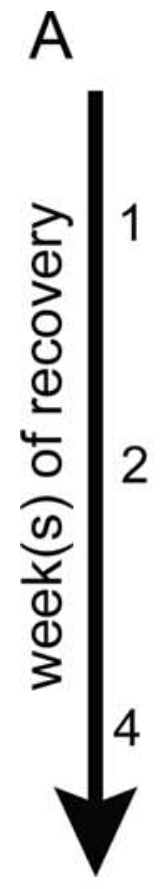

age-matched

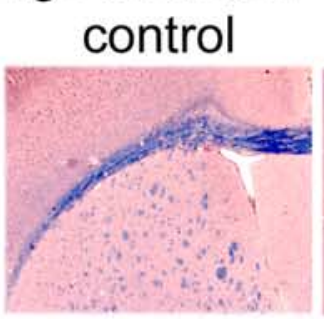
cuprizone

C

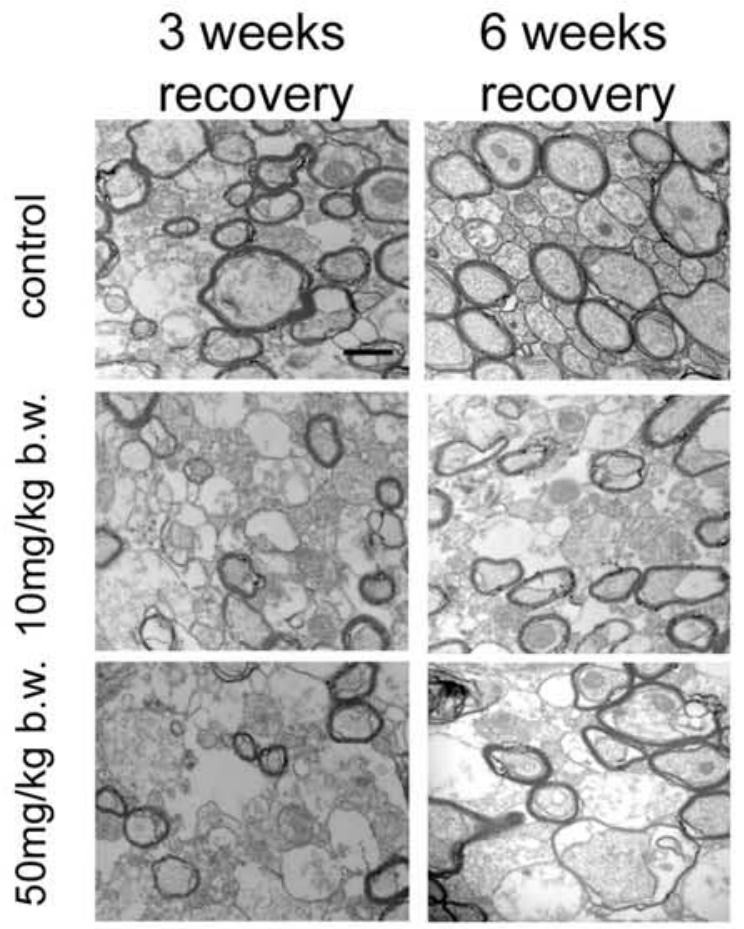

$1 \mathrm{mg} / \mathrm{kg}$ b.w. $10 \mathrm{mg} / \mathrm{kg}$ b.w. $50 \mathrm{mg} / \mathrm{kg}$ b.w.

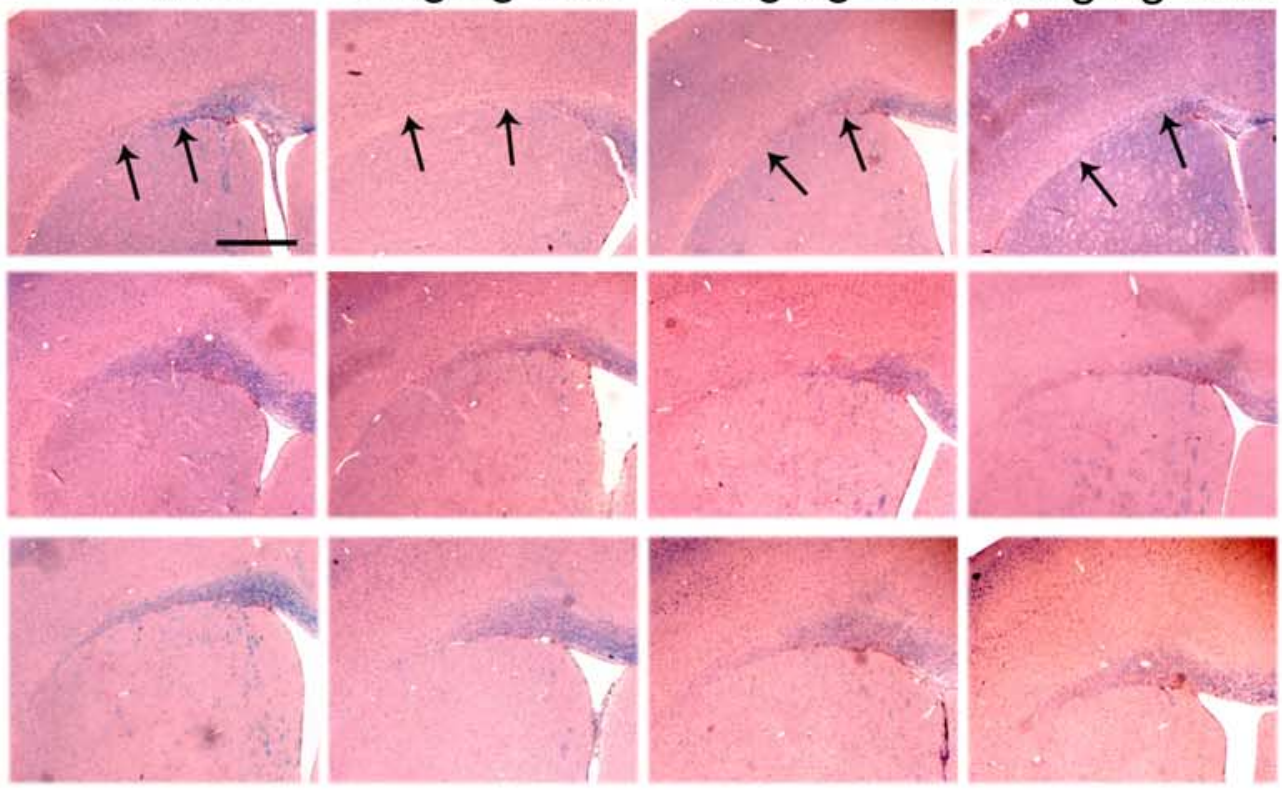

B
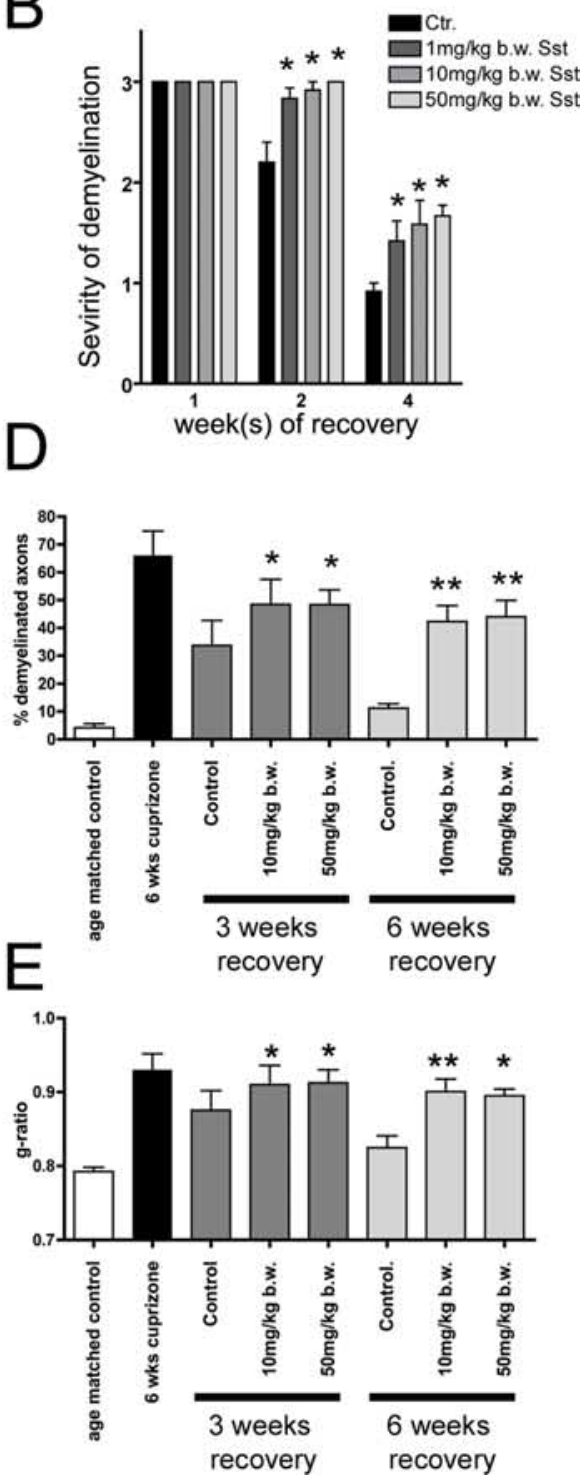
tures in simvastatin-treated animals during remyelination. However, additional direct statin-related effects on axonal integrity cannot be ruled out by our study and needs further clarification.

One important consideration is the applied dosage of Sst. In our study, the dosage range is broader than the approved dosage of Sst in humans. We have carefully chosen the doses to comply with previous reports that described a beneficial immunomodulatory effect in the inflammatory animal model of MS (Youssef et al., 2002; Aktas et al., 2003), studies which provided the basis for human trials for treatment with statins in MS (Sena et al., 2003; Vollmer et al., 2004). Furthermore, it is widely acknowledged that in rodents the metabolism of many drugs may vary considerably from humans, and therefore must be adjusted for application in humans. Nevertheless, we observed negative effects on remyelination at a dose of $1 \mathrm{mg} \mathrm{Sst} / \mathrm{kg}$ b.w., a dose which corresponds to the application used in trials of MS patients (Vollmer et al., 2004).

In this context, our study indicates that statins should be used carefully since long-term application may negatively influence the intrinsic remyelinating capacity, not only in MS patients, but also in other demyelinating diseases of the CNS.

\section{References}

Aktas O, Waiczies S, Smorodchenko A, Dorr J, Seeger B, Prozorovski T, Sallach S, Endres M, Brocke S, Nitsch R, Zipp F (2003) Treatment of relapsing paralysis in experimental encephalomyelitis by targeting Th1 cells through atorvastatin. J Exp Med 197:725-733.

Althaus HH (2004) Remyelination in multiple sclerosis: a new role for neurotrophins? Prog Brain Res 146:415-432.

Althaus HH, Hempel R, Klöppner S, Engel J, Schmidt-Schultz T, Kruska L, Heumann R (1997) Nerve growth factor signal transduction in mature pig oligodendrocytes. J Neurosci Res 50:729-742.

Barkhof F, Bruck W, De Groot CJ, Bergers E, Hulshof S, Geurts J, Polman CH, van der Valk P (2003) Remyelinated lesions in multiple sclerosis: magnetic resonance image appearance. Arch Neurol 60:1073-1081.

Bhat NR, Zhang P (1996) Activation of mitogen-activated protein kinases in oligodendrocytes. J Neurochem 66:1986-1994.

Blakemore WF (1973) Demyelination of the superior cerebellar peduncle in the mouse induced by cuprizone. J Neurol Sci 20:63-72.

Brück W, Kuhlmann T, Stadelmann C (2003) Remyelination in multiple sclerosis. J Neurol Sci 206:181-185.

Casey PJ (1995) Protein lipidation in cell signaling. Science 268:221-225.

Coetzee T, Fujita N, Dupree J, Shi R, Blight A, Suzuki K, Suzuki K, Popko B (1996) Myelination in the absence of galactocerebroside and sulfatide: normal structure with abnormal function and regional instability. Cell $86: 209-219$.

Franklin RJ (2002) Why does remyelination fail in multiple sclerosis? Nat Rev Neurosci 3:705-714.

Gensert JM, Goldman JE (1997) Endogenous progenitors remyelinate demyelinated axons in the adult CNS. Neuron 19:197-203.
Greenwood J, Steinman L, Zamvil SS (2006) Statin therapy and autoimmune disease: from protein prenylation to immunomodulation. Nat Rev Immunol 6:358-370.

Hiremath MM, Saito Y, Knapp GW, Ting JP, Suzuki K, Matsushima GK (1998) Microglial/macrophage accumulation during cuprizone-induced demyelination in C57BL/6 mice. J Neuroimmunol 92:38-49.

Irvine KA, Blakemore WF (2008) Remyelination protects axons from demyelination-associated axon degeneration. Brain 131:1464-1477.

Liang X, Draghi NA, Resh MD (2004) Signaling from integrins to Fyn to Rho family GTPases regulates morphologic differentiation of oligodendrocytes. J Neurosci 24:7140-7149.

Liao JK, Laufs U (2005) Pleiotropic effects of statins. Annu Rev Pharmacol Toxicol 45:89-118.

Ludwin SK (1978) Central nervous system demyelination and remyelination in the mouse: an ultrastructural study of cuprizone toxicity. Lab Invest 39:597-612.

Matsushima GK, Morell P (2001) The neurotoxicant, cuprizone, as a model to study demyelination and remyelination in the central nervous system. Brain Pathol 11:107-116.

Miron VE, Rajasekharan S, Jarjour AA, Zamvil SS, Kennedy TE, Antel JP (2007) Simvastatin regulates oligodendroglial process dynamics and survival. Glia 55:130-143.

Paintlia AS, Paintlia MK, Khan M, Vollmer T, Singh AK, Singh I (2005) HMG-CoA reductase inhibitor augments survival and differentiation of oligodendrocyte progenitors in animal model of multiple sclerosis. FASEB J 19:1407-1421

Paintlia AS, Paintlia MK, Singh AK, Singh I (2008) Inhibition of rho family functions by lovastatin promotes myelin repair in ameliorating experimental autoimmune encephalomyelitis. Mol Pharmacol 73:1381-1393.

Patrikios P, Stadelmann C, Kutzelnigg A, Rauschka H, Schmidbauer M, Laursen H, Sorensen PS, Brück W, Lucchinetti C, Lassmann H (2006) Remyelination is extensive in a subset of multiple sclerosis patients. Brain 129:3165-3172.

Riento K, Ridley AJ (2003) Rocks: multifunctional kinases in cell behaviour. Nat Rev Mol Cell Biol 4:446-456.

Saher G, Brügger B, Lappe-Siefke C, Möbius W, Tozawa R, Wehr MC, Wieland F, Ishibashi S, Nave KA (2005) High cholesterol level is essential for myelin membrane growth. Nat Neurosci 8:468-475.

Sena A, Pedrosa R, Graça Morais M (2003) Therapeutic potential of lovastatin in multiple sclerosis. J Neurol 250:754-755.

Stariha RL, Kikuchi S, Siow YL, Pelech SL, Kim M, Kim SU (1997) Role of extracellular signal-regulated protein kinases 1 and 2 in oligodendroglial process extension. J Neurochem 68:945-953.

Topol EJ (2004) Intensive statin therapy-a sea change in cardiovascular prevention. N Engl J Med 350:1562-1564.

Vollmer T, Key L, Durkalski V, Tyor W, Corboy J, Markovic-Plese S, Preiningerova J, Rizzo M, Singh I (2004) Oral simvastatin treatment in relapsing-remitting multiple sclerosis. Lancet 363:1607-1608.

Youssef S, Stüve O, Patarroyo JC, Ruiz PJ, Radosevich JL, Hur EM, Bravo M, Mitchell DJ, Sobel RA, Steinman L, Zamvil SS (2002) The HMG-CoA reductase inhibitor, atorvastatin, promotes a Th2 bias and reverses paralysis in central nervous system autoimmune disease. Nature 420:78-84. 